\title{
Constraining proton geometric fluctuations with incoherent diffractive vector meson production
}

\section{H. Mäntysaari*}

Physics Department, Brookhaven National Laboratory, Upton, NY 11973, USA

E-mail: mantysari@bnl.gov

\section{B. Schenke}

Physics Department, Brookhaven National Laboratory, Upton, NY 11973, USA

E-mail: bschenke@bnl.gov

\begin{abstract}
We calculate coherent and incoherent diffractive vector meson production at high energy including event-by-event fluctuations of the proton density profile. Large geometric fluctuations are found to be necessary in order to describe simultaneously both coherent and incoherent cross section measured at HERA. The color charge and saturation scale fluctuations are shown to have small effect on incoherent cross section at large momentum transfer, which leads to a conclusion of existence of strong event-by-event geometric fluctuations of the proton density profile.
\end{abstract}

XXIV International Workshop on Deep-Inelastic Scattering and Related Subjects

11-15 April, 2016

DESY Hamburg, Germany

${ }^{*}$ Speaker. 


\section{Introduction}

Deep inelastic scattering measurements performed at HERA have provided us an accurate picture of the partonic structure of the proton. In particular, the precise proton structure function measurements (see e.g. [1]) performed by $\mathrm{H} 1$ and ZEUS collaborations have revealed that the gluon densities grow rapidly when the momentum fraction $x$ carried by the gluon decreases. The quark and gluon densities - integrated over the transverse profile of the proton, have been obtained in very good accuracy over a wide kinematical range.

However, not much is known about the transverse structure of the proton and its event-byevent fluctuations. This knowledge is, first of all, of fundamental interest. In addition, initial state geometry can potentially have large impact on description of collective phenomena observed in high multiplicity proton-proton and proton-nucleus collisions (see Ref [2] for a review) using, for example, relativistic hydrodynamics, see e.g. Refs. [3, 4, 5].

Diffractive deep inelastic scattering is a powerful tool to obtain this information of the proton structure. Indeed, In the Good-Walker picture [6], diffraction is described in terms of states that diagonalize the scattering matrix. At high energy, these states are the ones where a virtual photon fluctuates into a quark-antiquark dipole, and with a particular configuration of the target. The cross section is obtained by averaging over the target configurations.

Performing the target average at the level of the scattering amplitude the target is required to remain intact (we call this coherent diffraction), and the cross section is sensitive to the average proton structure. On the other hand, averaging the cross section instead of the amplitude allows the target to break up, giving the total diffractive cross section. Subtracting the coherent contribution leaves us with only events where the target breaks up (called incoherent diffraction), which is then proportional to the variance of the target profile, see e.g. Refs. [7, 8, 9, 10].

In the high enery limit, a useful tool to describe the scattering process is obtained in the Color Glass Condensate (CGC) framework [11], which has been successfuly applied to describe, for example, deep inelastic scattering processes at small Bjorken- $x[12,13,14]$. Diffractive scattering is also naturally described within the CGC picture as we will show in Sec. 2. In our recent papers, we have shown how it is possible to describe the measured coherent and incoherent diffractive cross sections within the CGC picture $[15,16]$. A part of these results is summarized here.

\section{Diffractive deep inelastic scattering in the CGC framework}

Diffractive vector meson production can be naturally described within the CGC framework, where the scattering amplitude can be factorized as follows. First, the incoming virtual photon with virtuality $Q^{2}$ splits into a quark-antiquark pair with transverse separation $\mathbf{r}_{T}$ and quark carrying factor $z$ of the longitudinal momentum. This splitting is given in terms of the virtual photon wave function $\Psi\left(Q^{2}, z, r\right)$. Then the color dipole scatters off the hadronic target (in this work a proton) without exchanging a net color charge with dipole-target cross section $\sigma_{\text {dip }}^{\mathrm{p}}$. Finally, the dipole forms the final state system, in this work a vector meson. The $\gamma^{*} \rightarrow q \bar{q}$ splitting can be computed from perturbative QED, but formation of a bound state must be modeled. In this work, the Boosted- 
Gaussian wave function from Ref. [17] is used. The diffractive scattering amplitude reads [17]

$$
\begin{aligned}
A_{T, L}^{\gamma^{*} p \rightarrow J / \Psi p}\left(x_{\mathbb{P}}, Q^{2}, \Delta\right)=i \int \mathrm{d}^{2} \mathbf{r}_{T} \int \mathrm{d}^{2} \mathbf{b}_{T} \int \frac{\mathrm{d} z}{4 \pi} & \\
& \times\left(\Psi^{*} \Psi_{V}\right)_{T, L}\left(Q^{2}, \mathbf{r}_{T}, z\right) e^{-i\left[\mathbf{b}_{T}-(1-z) \mathbf{r}_{T}\right] \cdot \Delta} \frac{\mathrm{d} \sigma_{\mathrm{dip}}^{\mathrm{p}}}{\mathrm{d}^{2} \mathbf{b}_{T}}\left(\mathbf{b}_{T}, \mathbf{r}_{T}, x_{\mathbb{P}}\right) .
\end{aligned}
$$

Here $\mathbf{b}_{T}$ is the impact parameter and the the transverse momentum transfer is $\Delta$. The dipole-proton scattering amplitude is obtained from the IPsat or IP-Glasma parametrizations discussed in Sec. 3.

The coherent cross section is obtained by averaging Eq 2 over all the possible target proton configurations:

$$
\frac{\mathrm{d} \sigma^{\gamma^{*} p \rightarrow J / \Psi p}}{\mathrm{~d} t}=\frac{1}{16 \pi}\left|\left\langle A^{\gamma^{*} p \rightarrow J / \Psi p}\left(x_{\mathbb{P}}, Q^{2}, \Delta\right)\right\rangle\right|^{2},
$$

with $t \approx-\Delta^{2}$. When the target is required to break up, the incoherent cross section is obtained as a variance [7]

$$
\frac{\mathrm{d} \sigma^{\gamma^{*} p \rightarrow J / \Psi p^{*}}}{\mathrm{~d} t}=\frac{1}{16 \pi}\left(\left\langle\left|A^{\gamma^{*} p \rightarrow J / \Psi p}\left(x_{\mathbb{P}}, Q^{2}, \Delta\right)\right|^{2}\right\rangle-\left|\left\langle A^{\gamma^{*} p \rightarrow J / \Psi p}\left(x_{\mathbb{P}}, Q^{2}, \Delta\right)\right\rangle\right|^{2}\right) .
$$

Notice that the diffractive scattering amplitude (2) is proportional to the Fourier transfer of the transverse density profile of the proton. Thus, the coherent cross section probes directly the average density profile of the proton, and incoherent cross section (2.3) is sensitive to the event-by-event fluctuations of the density profile.

\section{Dipole-proton scattering}

In the diffractive scattering amplitude (2) all the QCD dynamics is enclosed in the dipole-target cross section $\sigma_{\text {dip }}^{\mathrm{p}}$. In this work, we study two different models for the dipole cross section.

The first model used here is the IPsat model, where the dipole-target cross section is written as

$$
\frac{\mathrm{d} \sigma_{\mathrm{dip}}^{\mathrm{p}}}{\mathrm{d}^{2} \mathbf{b}_{T}}\left(\mathbf{b}_{T}, \mathbf{r}_{T}, x_{\mathbb{P}}\right)=2\left[1-\exp \left(-\mathbf{r}^{2} F\left(x_{\mathbb{P}}, \mathbf{r}_{T}\right) T_{p}\left(\mathbf{b}_{T}\right)\right)\right] .
$$

Here $T_{p}\left(\mathbf{b}_{T}\right)$ is the proton spatial profile function which is assumed to be Gaussian, $T_{p}\left(\mathbf{b}_{T}\right)=$ $\frac{1}{2 \pi B_{p}} e^{-\mathbf{b}_{T}{ }^{2} /\left(2 B_{p}\right)}$. The function $F$ is proportional to the DGLAP evolved gluon distribution

$$
F\left(x_{\mathbb{P}}, \mathbf{r}_{T}^{2}\right)=\frac{\pi^{2}}{2 N_{\mathrm{c}}} \alpha_{\mathrm{s}}\left(\mu^{2}\right) x_{\mathbb{P}} g\left(x_{\mathbb{P}}, \mu^{2}\right),
$$

with $\mu^{2}=\mu_{0}^{2}+4 / \mathbf{r}_{T}^{2}$. The free parameters in this mode $\left(\mu_{0}^{2}, B_{p}\right.$ and initial condition for the DGLAP evolution of the gluon distribution $x g$ ) are obtained by performing a fit to HERA data in Ref. [13].

The geometric fluctuations are included in this model by assuming that the gluons in the proton are localized around the constituent quarks (or three hot spots) at points $\mathbf{b}_{T i}$. The positions of the constituent quarks are sampled from a Gaussian distribution with width $B_{q c}$. Then, the proton density profile is assumed to be Gaussian around the centers of the constituent quarks, and the 
width of these hot spots is denoted by $B_{q}$. Thus, the combination of $B_{q}$ and $B_{q c}$ sets the degree of geometric fluctuations in this picture. The fluctuations are included in the IPsat model by making a replacement $T_{p}\left(\mathbf{b}_{T}\right) \rightarrow \frac{1}{N_{q}} \sum_{i=1}^{N_{q}} T_{q}\left(\mathbf{b}_{T}-\mathbf{b}_{T i}\right)$ with $N_{q}=3$ and $T_{q}$ is Gaussian having a width $B_{q}$.

We also use the IP-Glasma framework that includes local color charge fluctuations and has been successfully used to calculate initial conditions for the hydrodynamical modeling of the heavy ion collisions. In this case the dipole-target cross section is obtained by first relating the color charge density locally to the saturation scale obtained from the IPsat model. Then, one solves the classical Yang-Mills equations and obtains the Wilson lines $V\left(\mathbf{x}_{T}\right)$ at each transverse point. The dipole-target cross section is then obtained from the Wilson lines as $\frac{\mathrm{d} \sigma_{\mathrm{dip}}^{\mathrm{p}}}{\mathrm{d}^{2} \mathbf{b}_{T}}\left(\mathbf{r}_{T}=\mathbf{x}_{T}-\mathbf{y}_{T}, \mathbf{b}_{T}=\left(\mathbf{x}_{T}+\right.\right.$ $\left.\left.\mathbf{y}_{T}\right) / 2, x_{\mathbb{P}}\right)=2\left(1-1 / N_{\mathrm{c}} \operatorname{Tr} V\left(\mathbf{x}_{T}\right) V^{\dagger}\left(\mathbf{y}_{T}\right)\right)$. The geometric fluctuations are included by using an IPsat model with geometric fluctuations to calculate saturation scale at every point on the transverse plane. For more details of the IP-Glasma model, we refer the reader e.g. to Refs. [18, 19].

\section{Results}



Figure 1: Coherent (thick lines) and incoherent(thin lines) diffractive $J / \Psi$ production at $\langle W\rangle=$ $75 \mathrm{GeV}$ calculated with strong (solid lines) and moderate (dashed) geometric fluctuations. The results are compared with $\mathrm{H} 1$ data [20]. The bands show statistical errors of the calculations.
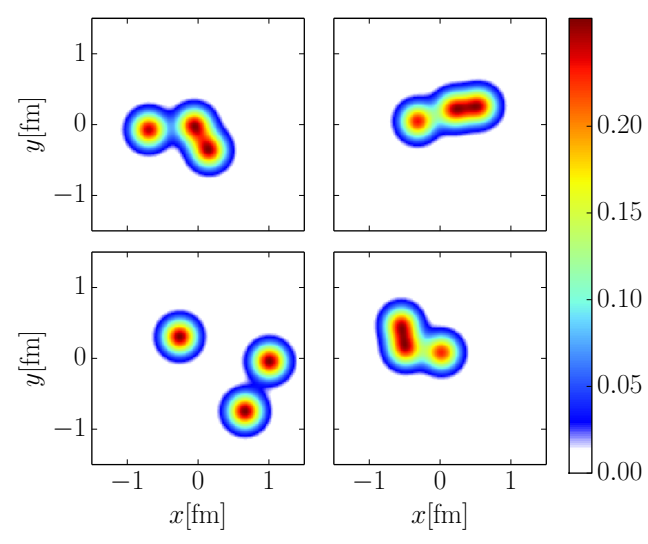

Figure 2: Examples of proton density profiles corresponding to proton with strong geometric fluctuations with parameters $B_{q}=3.3 \mathrm{GeV}^{-2}$ and $B_{q c}=$ $0.7 \mathrm{GeV}^{-2}$.

Coherent and incoherent diffractive $J / \Psi$ production at $\langle W\rangle=75 \mathrm{GeV}$ calculated using the IP-sat model is shown in Fig. 1. The results obtained using two different parametrizations for the proton structure: one with large event-by-event fluctuations (having $B_{q c}=3.3 \mathrm{GeV}^{-2} \gg B_{q}=$ $\left.0.7 \mathrm{GeV}^{-2}\right)$ and one which is relatively smooth $\left(B_{q c}=1.0 \mathrm{GeV}^{-2} \ll B_{q}=3.0 \mathrm{GeV}^{-2}\right)$. The results are compared with $\mathrm{H} 1$ data [20]. We find that the both of the two parametrizations give relatively good description fo the coherent HERA data, but the more smooth parametrization underestimates the incoherent cross section by several orders of magnitude. This suggests that large geometric event-by-event fluctuations are needed in order to describe incoherent vector meson production data. The amount of fluctuations is demonstrated in Fig. 2 where four example density profiles of the protons sampled from the more lumpy distribution are shown. 
Similarly the cross sections calculated using the IP-Glasma model are shown in Fig. 3. In this case, we show the result obtained without geometric fluctuations which is labeled as $B_{p}=$ $4.0 \mathrm{GeV}^{-2}$, which is the value obtained when fitting the IPsat model to coherent HERA data only [13]. Unlike in case of IPsat model, one also obtains nonzero incoherent cross section in that case due to color charge fluctuations. However, we see that color charge fluctuations alone are not enough to describe measured incoherent cross section.

The geometric fluctuations are then included by finding parameters $B_{q c}$ and $B_{q}$ that simultaneously give good description of the $\mathrm{H} 1$ data. The amount of fluctuations in that case is demonstrated by showing in Fig. 4 for a few example configurations the trace of the Wilson line at each transverse point, which is roughly proportional to the density of the proton. Again we find that large geometrical shape fluctuations are needed in order to describe the HERA data.

Finally we also include saturation scale fluctuations for each constituent quark separately following Ref. [21], where it is shown that proton-proton multiplicity fluctuations can be described within the IP-Glasma framework if $\ln Q_{s}^{2} /\left\langle Q_{s}^{2}\right\rangle$ fluctuations according to a Gaussian distribution whose width is $\sigma \approx 0.5$. We find that the saturation scale fluctuations mainly affect incoherent cross section at smallest $|t|$, and that at larger momentum transfer the dominant contribution comes from geometric fluctuations. This result is expected based on Ref. [7].

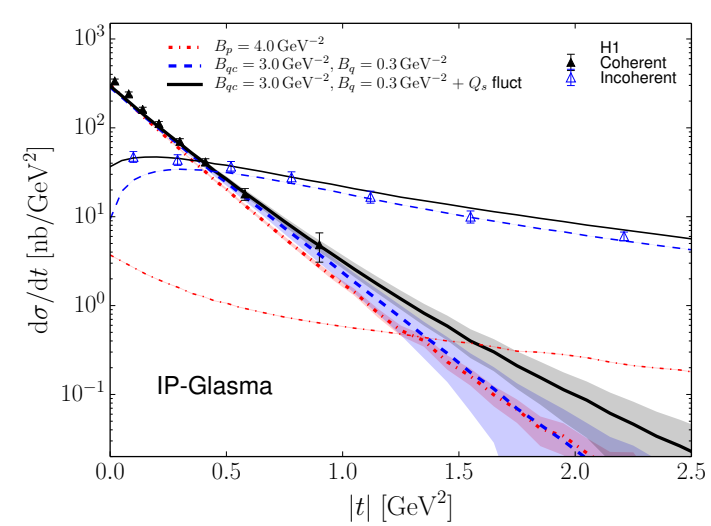

Figure 3: Coherent and incoherent diffractive $J / \Psi$ production at $\langle W\rangle=75 \mathrm{GeV}$ calculated from IPGlasma framework. The results are compared with H1 data [20]. The bands show statistical errors of the calculations.

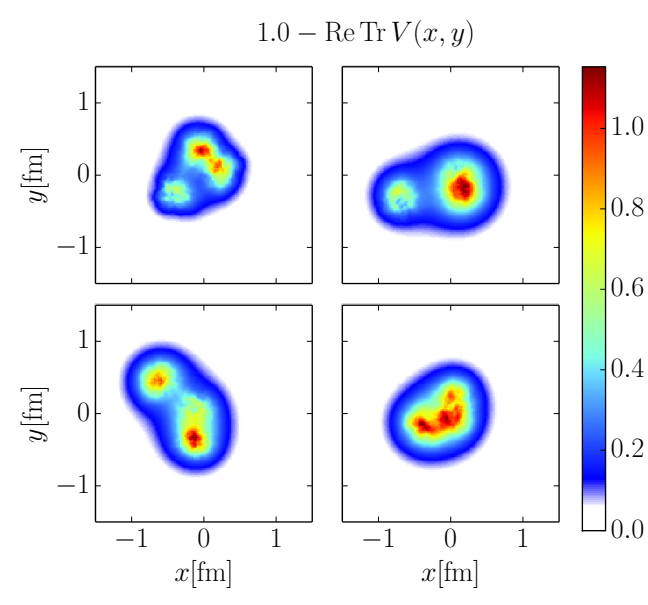

Figure 4: Examples of the proton density profile from the IP-Glasma framework. The quantity shown is $1.0-1.0 / N_{\mathrm{c}} \operatorname{Tr} V(x, y)$.

\section{Conclusions}

We have shown how diffractive vector meson production measured in deep inelastic scattering experiments at HERA can be used to constrain the amount of event-by-event fluctuations of the proton density. We find that strong geometric fluctuations are needed in order to obtain incoherent cross section compatible with the experimental data. The color charge fluctuations, on the other hand, are found to have small effect on the incoherent cross section. Similarly, the effect of saturation scale fluctuations on incoherent cross section is dominant only at small $|t|$. Our results can 
potentially have impact on hydrondynamical modeling of proton-nucleus collisions, which is left for future work.

\section{Acknowledgements}

This work was supported under DOE Contract No. DE-SC0012704. This research used resources of the National Energy Research Scientific Computing Center, which is supported by the Office of Science of the U.S. Department of Energy under Contract No. DE-AC02-05CH11231. BPS acknowledges a DOE Office of Science Early Career Award.

\section{References}

[1] H1 and ZEUS, H. Abramowicz et al., Eur. Phys. J. C75, 580 (2015), [arXiv:1506.06042 [hep-ex]].

[2] K. Dusling, W. Li and B. Schenke, Int. J. Mod. Phys. E25, 1630002 (2016), [arXiv:1509.07939 [nucl-ex]].

[3] C. E. Coleman-Smith and B. Müller, Phys. Rev. D89, 025019 (2014), [arXiv:1307.5911 [hep-ph]].

[4] B. Schenke and R. Venugopalan, Phys. Rev. Lett. 113, 102301 (2014), [arXiv:1405.3605 [nucl-th]].

[5] ATLAS, G. Aad et al., Eur. Phys. J. C76, 199 (2016), [arXiv:1508.00848 [hep-ex]].

[6] M. L. Good and W. D. Walker, Phys. Rev. 120, 1857 (1960).

[7] H. I. Miettinen and J. Pumplin, Phys. Rev. D18, 1696 (1978).

[8] L. Frankfurt, G. A. Miller and M. Strikman, Phys. Rev. Lett. 71, 2859 (1993), [arXiv:hep-ph/9309285 [hep-ph]].

[9] L. Frankfurt, M. Strikman, D. Treleani and C. Weiss, Phys. Rev. Lett. 101, 202003 (2008), [arXiv:0808.0182 [hep-ph]].

[10] A. Caldwell and H. Kowalski, Phys. Rev. C81, 025203 (2010), [arXiv:0909.1254].

[11] E. Iancu and R. Venugopalan, arXiv:hep-ph/0303204 [hep-ph].

[12] J. L. Albacete, N. Armesto, J. G. Milhano, P. Quiroga-Arias and C. A. Salgado, Eur. Phys. J. C71, 1705 (2011), [arXiv:1012.4408 [hep-ph]].

[13] A. H. Rezaeian, M. Siddikov, M. Van de Klundert and R. Venugopalan, Phys. Rev. D87, 034002 (2013), [arXiv:1212.2974].

[14] T. Lappi and H. Mäntysaari, Phys. Rev. D88, 114020 (2013), [arXiv:1309.6963 [hep-ph]].

[15] H. Mäntysaari and B. Schenke, arXiv:1603.04349 [hep-ph].

[16] H. Mäntysaari and B. Schenke, In preparation.

[17] H. Kowalski, L. Motyka and G. Watt, Phys. Rev. D74, 074016 (2006), [arXiv:hep-ph/0606272].

[18] B. Schenke, P. Tribedy and R. Venugopalan, Phys. Rev. Lett. 108, 252301 (2012), [arXiv:1202.6646 [nucl-th]].

[19] B. Schenke, P. Tribedy and R. Venugopalan, Phys. Rev. C86, 034908 (2012), [arXiv:1206.6805 [hep-ph]].

[20] H1, F. Aaron et al., JHEP 1005, 032 (2010), [arXiv:0910.5831 [hep-ex]].

[21] L. McLerran and P. Tribedy, Nucl. Phys. A945, 216 (2016), [arXiv:1508.03292 [hep-ph]]. 